\title{
Arginine deprivation therapy for malignant melanoma
}

\author{
Jung-Ki Yoon ${ }^{1,2}$ \\ Arthur E Frankel ${ }^{3}$ \\ Lynn G Feun ${ }^{4}$ \\ Suhendan Ekmekcioglu' \\ Kevin B Kim' \\ 'Department of Melanoma Medical \\ Oncology, The University of Texas MD \\ Anderson Cancer Center, Houston, \\ TX, USA; ${ }^{2}$ Hwasung Public Health \\ Center, Hwasung, South Korea, \\ ${ }^{3}$ Scott and White Cancer Research \\ Institute, Temple, TX, USA; ${ }^{4}$ Sylvester \\ Comprehensive Cancer Center, \\ University of Miami Miller School \\ of Medicine, Miami, FL, USA
}

This article was published in the following Dove Press journal:

Clinical Pharmacology:Advances and Applications

21 December 2012

Number of times this article has been viewed

\begin{abstract}
Despite recent development of promising immunotherapeutic and targeted drugs, prognosis in patients with advanced melanoma remains poor, and a cure for this disease remains elusive in most patients. The success of melanoma therapy depends on a better understanding of the biology of melanoma and development of drugs that effectively target the relevant genes or proteins essential for tumor cell survival. Melanoma cells frequently lack argininosuccinate synthetase, an essential enzyme for arginine synthesis, and as a result they become dependent on the availability of exogenous arginine. Accordingly, a therapeutic approach involving depletion of available arginine has been shown to be effective in preclinical studies. Early clinical studies have demonstrated sufficient antitumor activity to give rise to cautious optimism. In this article, the rationale for arginine deprivation therapy is discussed. Additionally, various strategies for depleting arginine are discussed and the preclinical and clinical investigations of arginine deprivation therapy in melanoma are reviewed.
\end{abstract}

Keywords: arginine deprivation, argininosuccinate synthetase, melanoma

\section{Introduction}

Melanoma results from malignant transformation of melanocytes. The incidence of malignant melanoma has steadily increased over the last several decades. ${ }^{1}$ In the US, an estimated 76,250 cases of melanoma will be diagnosed in 2012, and 9180 patients will die of this cancer during the same period. ${ }^{2}$ Fortunately, a majority of patients whose malignant melanoma is diagnosed early are cured with surgical intervention alone, and the 5 -year survival rate for cutaneous melanoma is approximately $93 \% .^{2}$ However, in a small subset of patients in whom melanoma cells escape the surgical excision and metastasize, a cure is very difficult due to the chemoresistant and radioresistant nature of melanoma cells. Patients who develop distant metastatic melanoma have a very poor prognosis, and the 5-year survival rate for patients with nonpulmonary visceral organ metastasis is approximately $10 \%{ }^{3}$

For patients whose melanoma lesion is not surgically resectable, systemic therapy is the treatment of choice. Despite tremendous effort over decades to improve systemic treatment, the clinical efficacy of the current systemic therapy for metastatic melanoma

Correspondence: Kevin B Kim Department of Melanoma Medical Oncology, The University of Texas MD Anderson Cancer Center, Unit 430, 1515 Holcombe Boulevard, Houston, TX 77030, USA

Tel + I 7137922921

Fax+l 7137451046

Email kkim@mdanderson.org is dismal, and the poor prognosis for this disease is linked directly to this lack of effective treatment. Dacarbazine is the only cytotoxic chemotherapy agent approved by the US Food and Drug Administration (FDA) for treatment of advanced melanoma. Its analog temozolomide has also been used widely for this disease; however, the response rates of these agents are less than $15 \% .{ }^{4}$ Clinical trials of combination chemotherapy failed to show significant overall survival advantage over dacarbazine alone. ${ }^{4}$ 
Because melanoma is an immunogenic tumor, immunotherapy has been considered a promising approach since the 1980s. High-dose interleukin-2 was approved by the FDA for its ability to induce durable clinical responses in advanced melanoma. ${ }^{5,6}$ However, less than $10 \%$ of patients who receive this intensive treatment experience meaningful durable clinical benefit. ${ }^{6}$ Recently, ipilimumab, an anticytotoxic T-lymphocyte antigen- 4 antibody, has received approval from the FDA and from the regulatory agency in Europe as a treatment for advanced melanoma on the basis of its survival advantage over the comparison treatment, the glycoprotein-100 peptide vaccine, in a secondline setting. ${ }^{7}$ Although a subset of the patients in that trial had a durable response lasting at least 2 years, the median overall survival of patients receiving ipilimumab with or without glycoprotein-100 peptide vaccine was only 10 months, and a majority of the patients ultimately died of the disease. ${ }^{7}$

Over the last 10 years, accumulating knowledge of the molecular signaling pathways in melanoma has led to the development of targeted therapy for the disease. About $50 \%-60 \%$ of melanomas harbor a $B R A F$ mutation, and about $80 \%-90 \%$ of $B R A F$-mutant melanomas have a single substitution mutation with glutamic acid replacing valine at the codon 600 position (V600E) in exon 15. This mutation activates the downstream mitogen-activated protein kinase kinase (MEK) and extracellular signal-related kinase-1/2 proteins and results in tumor cell proliferation and survival. ${ }^{8}$ In patients with advanced melanoma harboring this somatic mutation, vemurafenib (PLX4032; RG7204), a BRAF inhibitor, has been proven effective, with a response rate approaching 50\% and significant improvement in overall survival over dacarbazine, and was recently approved by the FDA for treatment of advanced melanoma harboring a BRAF mutation. ${ }^{9-11}$

Despite the initial success, this approach is effective only in patients whose melanoma harbors a $B R A F$ mutation, and even among these patients, a majority ultimately experience disease progression, with a median time to progression of approximately 6 months. ${ }^{9}$ Therefore, new strategies are still required to improve treatment outcomes. This review will discuss arginine deprivation as a possible relevant targeted therapy for metastatic melanoma.

\section{Overview of urea cycle and metabolic pathways related to arginine}

Arginine plays an important role in the production of proteins, nitric oxide, polyamines, nucleotides, proline, and glutamate. ${ }^{12}$ Arginine is produced from citrulline via the urea cycle in many cells including proximal renal tubule cells. ${ }^{12}$ The endogenous production of arginine is not sufficient for rapidly proliferating cells such as tumor cells, which require exogenous arginine to support their growth and survival. This dependency, called auxotrophy, may be exploited as an anticancer therapy by depleting exogenous support. It is known, for example, that acute lymphoblastic leukemia is auxotrophic for asparagine, a nonessential amino acid. L-asparaginase, which lowers plasma asparagine level, was proven to be an effective treatment for this leukemia. This example provides evidence for the utility of amino acid deprivation as therapy for cancer. ${ }^{13}$

In the urea cycle (Figure 1), arginine is synthesized from citrulline through the action of two tightly coupled enzymes, argininosuccinate synthetase (ASS) and argininosuccinate lyase. Arginine can be metabolized to ornithine by the enzyme arginase, and ornithine can be converted to citrulline by ornithine carbamoyltransferase (OTC) in the mitochondria. The citrulline can be utilized to synthesize arginine again.

Interestingly, many types of cancers, including melanoma, hepatocellular carcinoma (HCC), prostate cancer, and renal cell carcinoma (RCC), do not express the enzyme ASS, in contrast to most normal cells which do express the enzyme. ${ }^{14}$ Since ASS is an essential enzyme in the endogenous synthesis of arginine, tumor cells that lack ASS become more dependent on the availability of exogenous arginine. Therefore, ASSnegative tumor cells are particularly sensitive to arginine deprivation therapy.

\section{Overview of arginine deprivation therapy}

Arginine can be degraded by three enzymes: arginase, arginine decarboxylase, and arginine deiminase (ADI). These enzymes are potential biologic drugs for arginine deprivation therapy. Arginase is a naturally available human enzyme that is cytotoxic to tumor cells in vitro. However, arginase failed to show antitumor activity in mice with Taper liver cancer, ${ }^{15}$ likely because of its low affinity for arginine $\left(\mathrm{K}_{\mathrm{m}}=6 \mathrm{mmol} / \mathrm{L}\right.$ for native enzyme at physiologic $\left.\mathrm{pH}\right)$ and short half-life in the circulation (a few minutes).${ }^{16}$ Moreover, many normal tissues do not express OTC, ${ }^{17}$ which limits the synthesis of citrulline and, subsequently, arginine. Thus, abnormal accumulation of ornithine or loss of arginine in vital normal tissues can cause toxicity. ${ }^{18,19}$ A recombinant human arginase was developed that showed greater catalytic activity $\left(\mathrm{K}_{\mathrm{m}}=2.9 \mathrm{mmol} / \mathrm{L}\right)$ and prolonged in vivo half-life 


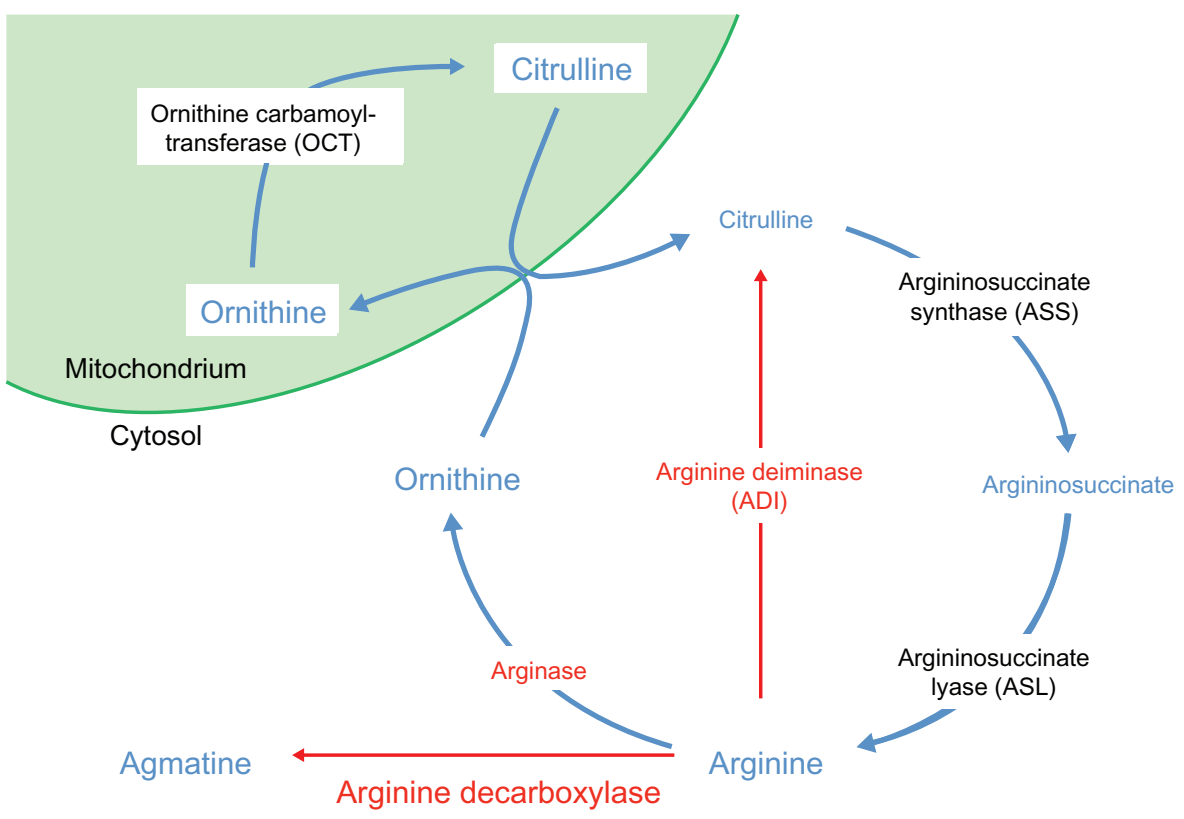

Figure I The urea cycle.

( $\sim 3$ days at physiologic $\mathrm{pH}$ ); this was accomplished by pegylation, linking the arginase covalently to polyethylene glycol (PEG). ${ }^{16}$ Currently, this agent is being investigated in an ongoing clinical trial for patients with HCC (NCI 01092091). A cobalt-substituted, pegylated recombinant human arginase (Co-ArgI-PEG) with significantly greater catalytic activity and stability is undergoing final testing and will be investigated in a clinical trial for solid tumor patients including melanoma (see below).

Arginine decarboxylase converts arginine to agmatine, and therefore it can be applied as a strategy to deprive cells of arginine. However, no known enzyme converts agmatine back to arginine, even in normal cells. Therefore, treatment with exogenous arginine decarboxylase is relatively toxic to normal cells, making this strategy unappealing. ${ }^{18}$

ADI converts arginine to citrulline and ammonia, the metabolites of the urea cycle (Figure 1). ${ }^{20}$ In contrast to arginase, ADI has a high affinity for arginine $\left(\mathrm{K}_{\mathrm{m}}=0.3 \mathrm{mmol} / \mathrm{L}\right),{ }^{21}$ and thus can more effectively lower plasma arginine level. However, ADI is not a human enzyme; it is found in other organisms, such as Mycoplasma spp. As a foreign protein, ADI from Mycoplasma spp. is immunogenic in humans. Introduced to human circulation, it has a short half-life and elicits neutralizing antibodies, but these shortcomings are mostly ameliorated by pegylation. Among various forms of pegylated ADI, ADI bound with PEG (molecular weight 20,000) via succinimidyl succinate (ADI-PEG20) has been found to be the optimal formulation. Pegylation increased the circulatory half-life of ADI from
4 hours to 7 days in mice, and induced only minimal antiADI immunoglobulin G..$^{22,23}$

\section{Preclinical studies of arginine deprivation therapy Pegylated ADI}

After demonstrating that infection of tumor cell lines with Mycoplasma spp. inhibited cell growth, investigators soon discovered that a protein in the infected cell supernatant had direct growth inhibitory activity. ${ }^{21,24}$ The protein was found to be ADI, and the tumor growth inhibition was dependent on depletion of arginine from the cell growth medium. The pegylated ADI-PEG20 formulation of ADI that was developed to reduce immunogenicity and prolong half-life was shown to retain its growth inhibitory effects in melanoma and $\mathrm{HCC}$ cell lines that lack messenger ribonucleic acid for ASS. Melanoma cell lines that had been transfected with human $A S S$ complementary DNA became resistant to ADI. ${ }^{22,23}$ In vivo studies using nude and severe combined immunodeficiency mouse xenograft models for melanoma and HCC demonstrated that ADI-PEG20 inhibited tumor growth and prolonged survival. In mice bearing a $\sim 0.5 \mathrm{~cm}$ human melanoma or HCC, injection of 5 IU of ADI-PEG20 once a week for 2 weeks caused tumor regression in 4 weeks, while tumor size increased continuously in mice injected with saline solution (controls). Furthermore, more than $50 \%$ of the mice treated with ADI-PEG20 survived at least 24 weeks, while all of the mice treated with saline solution died within 7 weeks. ${ }^{22}$ In a preclinical model using dogs with melanoma 
of the oral mucosa, 8 weeks of treatment with ADI-PEG20 yielded clinical benefit in six of ten (two complete responses, three partial responses, one stable disease). ${ }^{25}$

In cultured melanoma cells, treatment with ADI-PEG20 downregulates expression of hypoxia-inducible factor- $1 \alpha$ but upregulates expression of c-Myc. These results suggest that targeting tumor cell metabolism through modulation of HIF- $1 \alpha$ and c-Myc expression may improve the effectiveness of ADI-PEG20 in tumors that are auxotrophic for arginine. ${ }^{26}$

\section{Pegylated recombinant human arginase (rhArg[Mn]-PEG5000)}

To overcome the short circulatory half-life of arginase, $\operatorname{rhArg}(\mathrm{Mn})$-PEG5000 was developed and investigated with in vivo and in vitro studies in HCC. ${ }^{16} \mathrm{RhArg}(\mathrm{Mn})$-PEG5000 was effective in inhibiting growth of HCC cell lines as well as melanoma cell lines, with median inhibitory concentrations $\left(\mathrm{IC}_{50}\right)$ ranging from $0.1-2 \mathrm{IU} / \mathrm{mL}$. OTC-negative cancer cell lines were more sensitive to $\operatorname{rh} \operatorname{Arg}(\mathrm{Mn})$-PEG5000, while OTC-transfected cell lines were resistant. Since some OTC-negative cell lines that were sensitive to $\operatorname{rh} \operatorname{Arg}(\mathrm{Mn})$ PEG5000 had ASS expression, this arginase may be an alternative for the cancer patient with an ASS-expressing tumor.

This agent was tested in nude mice bearing OTCdeficient human HCC xenografts. Mice were treated once a week for 2 months with $250 \mathrm{IU}$ of $\operatorname{rhArg}(\mathrm{Mn})$-PEG5000 or saline solution. In contrast to the control group, which showed progressive tumor growth, the treatment group showed tumor-retarding activity. In experiments in which human melanomas and mouse melanomas were implanted into mice and treated twice weekly with low (200 IU) or high (400 IU) doses of rhArg(Mn)-PEG5000 or with saline solution (controls), the tumor growth of animals treated with $\operatorname{rhArg}(\mathrm{Mn})$-PEG5000 was suppressed $>50 \%$ relative to that of controls. ${ }^{27}$

\section{Cobalt-substituted human arginase}

To improve catalytic activity of arginase, cobalt $\left(\mathrm{Co}^{2+}\right)$ was substituted for manganese $\left(\mathrm{Mn}^{2+}\right)$ in recombinant human arginase $\mathrm{I}(\mathrm{rhArgI}[\mathrm{Co}])$. This enzyme showed ten-fold higher catalytic activity $\left(\mathrm{k}_{\mathrm{cat}} / \mathrm{K}_{\mathrm{m}}\right)$ than $\operatorname{rh} \operatorname{Arg}(\mathrm{Mn})$, and the logarithmic measure of the acid dissociation constant $\left(\mathrm{pK}_{\mathrm{a}}\right)$ shifted from 8.5 to 7.5. In vitro cytotoxic studies in multiple cell lines showed $\mathrm{IC}_{50}$ values for $\operatorname{rhArgI(Co)-PEG5000~that~were~12-15-fold~lower~}$ than those of $\operatorname{rhArgI}(\mathrm{Mn}) .{ }^{28} \mathrm{In}$ in vivo studies, this chemically engineered arginase was pegylated (rhArgI-[Co]-PEG5000) and injected once or twice a week at a dose of $8-10 \mathrm{mg} / \mathrm{kg}$ in normal mice and mice bearing a human HepG2 (HCC) or Panc-1 (pancreatic cancer) tumor xenograft. ${ }^{29}$ Treatment of normal mice with doses exceeding $10 \mathrm{mg} / \mathrm{kg}$ weekly led to inappetance, dehydration, marrow necrosis, and death. Weekly injection of $8 \mathrm{mg} / \mathrm{kg}$ induced regression in tumor xenografts, with tumor cells expressing activated caspase-3. ${ }^{29}$ However, rhArgI-(Co)-PEG5000 also causes cytotoxicity in normal cells which do not express OTC and thus cannot synthesize citrulline. Recently, supplementation of L-citrulline (0.052-38 mmol/L) was shown to rescue normal cells from the cytotoxic effect of rhArgI-(Co)-PEG5000 in vitro, ${ }^{17}$ suggesting that this strategy can possibly improve the therapeutic index of rhArgI-(Co)-PEG5000 treatment.

\section{Overcoming resistance to arginine deprivation therapy}

In addition to the efforts to improve enzymatic properties of arginine depriving enzymes, some studies have focused on methods to overcome resistance to arginine deprivation therapy. There are at least two mechanisms by which ASSdeficient cells develop resistance to arginine deprivation. ${ }^{18}$ The first entails tumor cell autophagy to generate excess intracellular arginine. In the stress environment, cells can suffer apoptosis or can evade apoptosis for certain periods with autophagy - the degradation of intracellular components through lysosomal machinery. After prolonged arginine deprivation, tumors have a tendency to undergo autophagy rather than apoptosis. Therefore, agents that inhibit autophagy can be combined with arginine deprivation to potentially increase antitumor activity in melanoma.

The second mechanism is upregulation of the ASS enzyme to facilitate arginine synthesis in tumor cells and reverse arginine auxotrophy. It has been shown that the ASS-deficient melanomas of two patients who were treated with ADI-PEG20 had upregulation of ASS protein and ASS messenger ribonucleic acid at the time of disease progression. ${ }^{30,31}$ One approach to counter the resistance is to combine agents with different mechanisms of action to induce a higher degree of tumor apoptosis and thus delay the development of resistance. Another potential strategy is to inhibit ASS expression by using a pharmacological approach. ${ }^{32}$

In an attempt to combine arginine deprivation therapy with cytotoxic drugs, the antitumor activity of the combination of ADI-PEG20 $(0.05 \mu \mathrm{g} / \mathrm{mL})$ and cisplatin $(0.1 \mu \mathrm{g} / \mathrm{mL})$ was investigated in melanoma cell lines. ${ }^{33}$ Growth inhibition was synergistic with the combination in comparison to ADI-PEG20 or cisplatin alone. 
Arginine deprivation was shown recently to induce activation of the mitogen-activated protein kinase pathway. In experimental arginine deprivation treatment in the Mel1220 cell line, upregulation of phosphorylated MEK and extracellular signal-related kinase- $1 / 2$ proteins, but no significant changes in BRAF kinase, was observed following treatment with ADI-PEG20. ${ }^{18}$ When a MEK inhibitor (U0126) was combined with ADI-PEG20 treatment, both growth inhibition and apoptosis were increased..$^{18}$ The combination treatment inhibited growth by $80 \%$, while ADIPEG20 alone and U0126 alone inhibited growth by $15 \%$ and $25 \%$, respectively. These findings suggest that melanoma cells may depend on the mitogen-activated protein kinase signaling pathway to survive upon arginine deprivation, and addition of a MEK inhibitor to arginine deprivation therapy may inhibit or delay the resistance.

Combination of ADI-PEG20 with an apoptosis-inducing agent, tumor necrosis factor-related apoptosis-inducing ligand (TRAIL), might also be useful. ADI-PEG20 induces expression of death receptor- $4 / 5$, a cell surface receptor for TRAIL, and has an additive effect with the antitumor activity of TRAIL. When the combination of ADI-PEG20 $(0.1 \mu \mathrm{g} / \mathrm{mL})$ and recombinant TRAIL $(100 \mathrm{ng} / \mathrm{mL})$ was tested in melanoma cell lines (SK-Mel-2, A375, Mel-1220, A2058), ADI-PEG20 alone inhibited growth by $50 \%-75 \%$, and TRAIL alone by $15 \%-25 \%$. In comparison, the combination inhibited growth by $>80 \%$. In addition, while ADI-PEG20 or TRAIL alone results in $0 \%-25 \%$ cell death, the combination of these two agents increased cell death to $70 \% \cdot{ }^{34}$ Coadministration of ADIPEG20 with canavanine, an arginine analog of plant origin, accelerated apoptosis induced by arginine deprivation. ${ }^{35}$ The $\mathrm{IC}_{50}$ of canavanine in arginine-rich culture medium was 3-10 $\mathrm{mmol} / \mathrm{L}$, and the $\mathrm{IC}_{50}$ in arginine-free culture medium was $0.03-0.07 \mathrm{mmol} / \mathrm{L}$. It was proposed that canavanine is incorporated into protein synthesis in place of arginine in arginine-deprived conditions, resulting in the synthesis of nonfunctional proteins and causing cell death. Interestingly, canavanine also sensitizes human tumor cells to irradiation in arginine-depleted condition. ${ }^{36}$ Low-dose canavanine has a prominent synergistic effect on arginine deprivation-induced radiosensitization. These findings suggest that a new approach combining antimetabolic therapy and chemoradiation may be a promising therapeutic strategy.

\section{Arginine deprivation therapy in other cancers}

Arginine deprivation has been shown to be a promising treatment option not only for melanoma and $\mathrm{HCC}$, but also for other arginine auxotrophic cancers, including malignant pleural mesothelioma (MPM), ${ }^{37} \mathrm{RCC},{ }^{38}$ prostate cancer, ${ }^{39}$ human T-lymphoblastic leukemia, ${ }^{40}$ and osteosarcoma. ${ }^{41}$ Fifty-two (63\%) of 82 MPM tissues from patients showed reduced or absent ASS protein expression. ASS-negative MPM cell lines failed to grow in arginine-depleted culture medium, while the same cell lines grew in argininecontaining culture medium; ASS-positive cell lines grew in either medium. ${ }^{37}$

Tumor samples from 98 patients with RCC were examined by immunohistochemistry and were found to be devoid of ASS expression. The growth of an RCC cell line (RenCa) was inhibited by ADI ( $0.005 \mathrm{IU} / \mathrm{mL}$ for 72 hours) to $20 \%-40 \%$ that of controls. When RCC-bearing mice were treated with ADI (1-5 IU/day), the tumors showed dose-dependent regression in size and weight. Furthermore, all treated mice survived for more than 90 days after tumor implantation, while all control mice died between 40-72 days after tumor implantation. ${ }^{38}$

Immunohistochemical analysis of 88 human prostate tumor specimens and 59 normal prostate tissues showed that none of tumor tissues expressed ASS, while $27 \%$ of normal tissue samples expressed some degree of ASS. Apoptosis was induced in ASS-negative prostate carcinoma cell lines (LNCaP and PC3) by $0.3 \mu \mathrm{g} / \mathrm{mL}$ ADI-PEG20. In a mouse xenograft model of human prostate cancer, mice were treated with weekly injection of ADI-PEG20 (5 IU) or a combination of ADI-PEG20 and docetaxel $(10 \mathrm{mg} / \mathrm{kg})$. Tumor volumes from the treated group were approximately seven times smaller than those from the control group, and a synergistic effect with the docetaxel was observed. ${ }^{39}$

Pulmonary metastasis is the most significant prognostic determinant for osteosarcoma. Global gene expressions of 19 patients who received surgical resection with neoadjuvant chemotherapy were analyzed. Absence of ASS expression was shown to be a predictive marker for development of pulmonary metastasis. ${ }^{41}$ However, ASS-negative osteosarcoma cells were auxotrophic for arginine, and cell cycle arrest occurred in arginine-depleting conditions, as in other ASS-negative cancer cells. ${ }^{41}$ Therefore, absence of ASS expression may be a potential target for arginine deprivation therapy in patients with osteosarcoma.

\section{Clinical trials of arginine deprivation therapy (Table I)}

The first case of a patient treated with ADI-PEG20 was presented in $2003 .{ }^{42}$ The patient, who had idiopathic cirrhosis and unresectable HCC, was treated with escalating 
doses of ADI-PEG20. At a dose of $160 \mathrm{IU} / \mathrm{m}^{2}$, the serum $\alpha$-fetoprotein level was decreased and the tumor was reduced in size to allow surgical treatment, while the patient suffered no significant adverse events. This report led to a Phase I/II trial $(n=19)$ of ADI-PEG20 in patients with nonresectable HCC. ${ }^{43}$ The optimum biological dose of ADI-PEG20 injected intramuscularly was $160 \mathrm{IU} / \mathrm{m}^{2}$, which lowered the plasma arginine concentration to an undetectable level $(<2 \mu \mathrm{mol} / \mathrm{L})$ for more than 7 days. The response rate was $47 \%$ (two complete responses and seven partial responses), and the rate of stable disease was $37 \%$ among 19 enrolled patients. The median survival was 410 days, and no significant toxicity was observed. There was no grade 4 toxicity and the only grade 3 adverse events were the elevated serum lipase and amylase level in one patient each.

In a two-cohort, dose-escalation Phase I/II study of ADI-PEG20 in patients with metastatic melanoma, the optimum biological dose to reduce the blood arginine level to a nondetectable level $(<2 \mathrm{mmol} / \mathrm{L})$ for at least 7 days was determined to be $160 \mathrm{IU} / \mathrm{m}^{2} /$ week. $^{44}$ There were no grade 3-4 toxic effects directly attributable to the drug. Among six patients treated with $640 \mathrm{IU} / \mathrm{m}^{2}$ of ADI-PEG20 via intramuscular injection once a week, no significant toxic effects were noted except grade 1 injection site pain. Other common adverse events were the elevated serum amylase, lipase, and transaminases, and hypotension, which were all grade $1-2$. The maximum tolerated dose was not reached at $640 \mathrm{IU} / \mathrm{m}^{2} /$ week. Six $(25 \%)$ of 24 patients showed a response to treatment (five partial responses and one complete response), and an additional six patients (25\%) had stable disease of at least 3 months duration. All these patients received ADI-PEG20 at a dose of $160 \mathrm{IU} / \mathrm{m}^{2}$ or higher. The median overall survival duration was 15 months in these patients with stage IV disease. None of the plasma samples from any of the 39 patients showed measurable enzyme-neutralizing activity.

In another Phase II study of ADI-PEG20 in patients with melanoma, ${ }^{30} 36$ patients were treated weekly with ADI-PEG20 at 160-320 IU/m² intramuscularly. Ten (28\%) of the patients had a clinical benefit (partial response, minor response, or stable disease). Including mixed response, 14 (39\%) of 36 patients showed antitumor effects. Twenty patients whose disease showed no response to $160 \mathrm{IU} / \mathrm{m}^{2} /$ week were treated with $320 \mathrm{IU} / \mathrm{m}^{2} /$ week, and four of them had a response. This result suggests that $320 \mathrm{IU} / \mathrm{m}^{2} /$ week is the appropriate dosage for future trials. This dose was well tolerated, but further dose escalation is limited by the large volume of intramuscular injection of ADI-PEG20. Pretreatment tumor samples from 26 patients were analyzed for ASS expression. Interestingly, among the 16 patients whose tumors did not express ASS, three $(29 \%)$ had a clinical response and eight (50\%) had some clinical benefit (clinical response or stable disease). In contrast, only one of ten patients whose tumor expressed ASS had clinical benefit $(P=0.01)$. This finding suggests that ASS expression status, which can be determined either

Table I Clinical trials of arginine deprivation therapy in cancer

\begin{tabular}{|c|c|c|c|c|c|}
\hline Agents & Cancer types & Phase & Clinical efficacy & Common toxicity & Reference \\
\hline \multirow[t]{16}{*}{ ADI-PEG20 } & \multirow[t]{3}{*}{ MM } & \multirow[t]{3}{*}{$1 / I I$} & OR: $6 / 24(25 \%)$ & Injection site pain, elevated & \multirow[t]{3}{*}{44} \\
\hline & & & SD: $6 / 24(25 \%)$ & serum amylase, lipase, and & \\
\hline & & & & transaminases, hypotension & \\
\hline & MM & II & OR + SD: $10 / 36(28 \%)$ & Discomfort at injection site & 30 \\
\hline & \multirow[t]{2}{*}{$\mathrm{HCC}$} & \multirow[t]{2}{*}{$\mathrm{I} / \mathrm{II}$} & OR: $9 / 19$ (47\%) & Elevated serum lipase, amylase, & \multirow[t]{2}{*}{43} \\
\hline & & & SD: $7 / 19$ (37\%) & bilirubin, creatinine, uric acid & \\
\hline & \multirow[t]{4}{*}{$\mathrm{HCC}$} & \multirow[t]{4}{*}{ II } & OR: $2 / 76(3 \%)$ & Injection site discomfort, fever, & \multirow[t]{4}{*}{48} \\
\hline & & & SD: $50 / 76(61 \%)$ & anemia, abnormal serum sodium & \\
\hline & & & & and potassium levels, decreased & \\
\hline & & & & fibrinogen & \\
\hline & \multirow[t]{3}{*}{$\mathrm{HCC}$} & \multirow[t]{3}{*}{ II } & OR: 0/7I (0\%) & \multirow{6}{*}{$\begin{array}{l}\text { Local and/or allergic reactions, } \\
\text { hyperuricemia, pruritus, fatigue, } \\
\text { hyperammonemia, fever, diarrhea }\end{array}$} & \multirow[t]{6}{*}{49} \\
\hline & & & SD: $22 / 7 \mid(31 \%)$ & & \\
\hline & & & & & \\
\hline & $\mathrm{HCC}$ & III & Ongoing - NCTOI 287585 & & \\
\hline & ASS(-) MPM & ॥ & Ongoing - NCTOI 279967 & & \\
\hline & SCLC & $\|$ & Ongoing - NCT0I2660I8 & & \\
\hline \multirow[t]{3}{*}{ rhArgl(Mn)-PEG5000 } & \multirow[t]{3}{*}{$\mathrm{HCC}$} & \multirow[t]{3}{*}{ I } & \multirow[t]{3}{*}{ SD: $4 / 8(50 \%)$} & Diarrhea, nausea, abdominal pain, & \multirow[t]{3}{*}{50} \\
\hline & & & & liver dysfunction, serum bilirubin & \\
\hline & & & & elevation & \\
\hline
\end{tabular}

Abbreviations: ASS, argininosuccinate synthetase; HCC, hepatocellular carcinoma; MM, metastatic melanoma; MPM, malignant pleural mesothelioma; OR, overall response (complete + partial response); SCLC, small cell lung carcinoma; SD, stable disease. 
by immunohistochemical staining or reverse-transcription polymerase chain reaction analysis for messenger ribonucleic acid expression may be a useful predictive marker for ADI-PEG20 treatment efficacy. ${ }^{14,30}$

It must be noted that no grade 3-4 toxic effects could be directly attributed to ADI-PEG20 in melanoma patients. ${ }^{30,44}$ Therefore, this drug is likely be combined safely with other treatment modalities, such as chemotherapeutic or immunotherapeutic drugs.

ADI-PEG20 treatment also resulted in a dose-dependent decrease of the plasma level of nitric oxide, ${ }^{44}$ which is biosynthesized by inducible nitric oxide synthase and exerts growth-promoting and proangiogenic effects in tumors, including melanoma. ${ }^{45-47}$ This finding suggests that one of the mechanisms of action of ADI-PEG20, and possibly other arginine deprivation therapies, is inhibition of nitric oxide synthesis.

ADI-PEG20 is being tested in a clinical trial setting in other cancer types. After the first Phase I/II trial of ADIPEG20 in patients with unresectable HCC, two parallel Phase II studies were conducted. In one trial, 80 patients who had unresectable metastatic HCC were treated with $80 \mathrm{IU} / \mathrm{m}^{2}$ or $160 \mathrm{IU} / \mathrm{m}^{2}$ of ADI-PEG20 weekly for up to 6 months. ${ }^{48}$ This trial yielded an objective response rate of $2.6 \%$ and a disease control rate (which included complete and partial responses and stable disease) of $65 \%$. The median overall survival was 11.4 months. Two patients $(2.6 \%)$ were withdrawn from the study because of immunogenic-related adverse events. Most toxic effects were grade 1-2 (with some grade 3), and there were no deaths attributed to ADI-PEG20. The common side effects were skin irritation or discomfort at the site of injection, fever, anemia, abnormal serum sodium and potassium levels, and decreased fibrinogen. Although there was grade 1-2 transient and reversible encephalopathy, it was not clear if the encephalopathy was related to the drug or the preexisting hepatic dysfunction. After approximately 60 days of treatment, the antibody titer of ADI-PEG20 was steadily increased, and serum arginine levels returned to baseline level. ${ }^{48}$ In the other Phase II study, 71 Asian patients with advanced HCC received weekly intramuscular injection of ADI-PEG20 at doses of $160 \mathrm{IU} / \mathrm{m}^{2}$ or $320 \mathrm{IU} / \mathrm{m}^{2}{ }^{49}$ There were no complete or partial responses. The disease control rate was $31 \%$, and the median overall survival was 7.3 months. The major treatment-related adverse events were grade 1-2 local and/or allergic reactions, hyperuricemia, pruritus, fatigue, hyperammonemia, fever, and diarrhea. ${ }^{49}$

A randomized double-blind Phase III trial of ADI-PEG20 in patients with advanced HCC is now underway
(NCT 01287585). Phase II studies are ongoing in patients with ASS-negative MPM (NCT 01279967) and in patients with relapsed small-cell lung cancer (NCT 01266018).

Results of a Phase I study of recombinant human arginase I (rhArgI[Mn]-PEG5000) in 15 patients with advanced HCC were recently reported. ${ }^{50}$ Weekly doses of rhArgI(Mn)-PEG5000 ranged from 500-3500 IU/kg, and the maximum tolerated dose was determined to be $1600 \mathrm{IU} / \mathrm{kg}$. The most common adverse effect was diarrhea (14\%), and the dose-limiting toxic effect was grade 3 prolonged elevation of serum bilirubin in two patients who received doses of $2500 \mathrm{IU} / \mathrm{kg}$. Among eight patients who were evaluated for tumor response, four $(50 \%)$ had stable disease for more than 8 weeks. The median time to progression was 2.8 months, and the median overall survival was 5.1 months.

Co-ArgI-PEG has been produced under good manufacturing practice for a Phase I clinical study in advanced cancer (including melanoma) patients in the fall of 2012 (Frankel, unpublished data, 2012).

\section{Conclusion}

Despite recent advances in melanoma therapy, a majority of patients with this disease will ultimately die of it, and a new approach to systemic therapy is urgently needed. Because melanoma cells with a low level of ASS enzyme become dependent on exogenous arginine for their proliferation and survival, arginine deprivation therapy appears to be a promising novel approach to melanoma therapy without significant toxicity to normal organs. There are several advantages of the arginine deprivation therapy in comparison to the conventional cytotoxic chemotherapy. As the clinical benefit of arginine deprivation therapy is likely dependent on the level of ASS enzyme, it is possible to select appropriate patients who are likely to respond to the therapy by analyzing the tumoral expression level of ASS. In addition, the investigation of a number of the arginine deprivation therapy drugs have shown a favorable safety profile, with less life-threatening adverse events, such as severe myelosuppression.

However, there are disadvantages and challenges of this therapeutic approach. The arginine deprivation therapy is not likely to be useful in patients with melanomas with a high ASS expression level, limiting the potential candidates for this therapy. Furthermore, the arginine deprivation therapy to date has yet to show the high response rate with durable tumor control in patients with melanoma. Currently, newer generation drugs are being tested clinically, and some of the newer drugs, such as ADI-PEG20, have shown 
promising clinical activity in early-phase clinical studies. It is the authors' hope that these drugs significantly improve the clinical benefit in patients in the near future. It is also possible that the arginine deprivation therapy potentiates the clinical efficacy of cytotoxic agents or targeted therapy drugs, as shown in the preclinical studies. Because of its good tolerability as a single agent, it will be easier to combine arginine deprivation therapy with other drugs.

Successful clinical investigation of arginine deprivation therapy will require selection of patients with the relevant biomarker (ie, lack of ASS expression in the tumor lesions), combination of arginine depriving agents with other additive or synergistic agents, and development of more potent drugs with more favorable pharmacokinetic profiles.

\section{Acknowledgment}

The authors thank Ms Kathryn Hale for her editorial assistance.

\section{Disclosure}

The authors report no conflicts of interest in this work.

\section{References}

1. Weinstock MA. Epidemiology, etiology, and control of melanoma. Med Health R I. 2001;84(7):234-236.

2. Siegel R, Naishadham D, Jemal A. Cancer statistics, 2012. CA Cancer J Clin. 2012;62(1):10-29.

3. Balch CM, Gershenwald JE, Soong SJ, et al. Final version of 2009 AJCC melanoma staging and classification. J Clin Oncol. 2009;27(36): 6199-6206.

4. Tsao H, Atkins MB, Sober AJ. Management of cutaneous melanoma. N Engl J Med. 2004;351(10):998-1012.

5. Rosenberg SA, Lotze MT, Yang JC, et al. Experience with the use of high-dose interleukin-2 in the treatment of 652 cancer patients. Ann Surg. 1989;210(4):474-484.

6. Atkins MB, Lotze MT, Dutcher JP, et al. High-dose recombinant interleukin 2 therapy for patients with metastatic melanoma: analysis of 270 patients treated between 1985 and 1993. J Clin Oncol. 1999;17(7): 2105-2116.

7. Hodi FS, O’Day SJ, McDermott DF, et al. Improved survival with ipilimumab in patients with metastatic melanoma. $N$ Engl $J$ Med. 2010;363(8):711-723.

8. Flaherty KT, McArthur G. BRAF, a target in melanoma: implications for solid tumor drug development. Cancer. 2010;116(21):4902-4913.

9. Flaherty KT, Puzanov I, Kim KB, et al. Inhibition of mutated, activated BRAF in metastatic melanoma. $N$ Engl J Med. 2010;363(9):809-819.

10. Ribas A, Kim KB, Schuchter LM, et al. BRIM-2: an open-label, multicenter phase II study of vemurafenib in previously treated patients with BRAF V600E mutation-positive metastatic melanoma [abstract]. J Clin Oncol. 2011;29:8509.

11. Chapman $\mathrm{PB}$, Hauschild $\mathrm{A}$, Robert $\mathrm{C}$, et al. Improved survival with vemurafenib in melanoma with BRAF V600E mutation. N Engl J Med. 2011;364(26):2507-2516.

12. Morris SM Jr. Arginine: beyond protein. Am J Clin Nutr. 2006;83(2): 508S-512S.

13. Muller HJ, Boos J. Use of L-asparaginase in childhood ALL. Crit Rev Oncol Hematol. 1998;28(2):97-113.
14. Dillon BJ, Prieto VG, Curley SA, et al. Incidence and distribution of argininosuccinate synthetase deficiency in human cancers: a method for identifying cancers sensitive to arginine deprivation. Cancer. 2004;100(4):826-833.

15. Savoca KV, Davis FF, van Es T, McCoy JR, Placzuk NC. Cancer therapy with chemically modified enzymes. II. The therapeutic effectiveness of arginase, and arginase modified by the covalent attachment of polyethylene glycol, on the taper liver tumor and the L5178Y murine leukemia. Cancer Biochem Biophys. 1984;7(3):261-268.

16. Cheng PN, Lam TL, Lam WM, et al. Pegylated recombinant human arginase (rhArg-peg5,000 $\mathrm{mw}$ ) inhibits the in vitro and in vivo proliferation of human hepatocellular carcinoma through arginine depletion. Cancer Res. 2007;67(1):309-317.

17. Agrawal V, Woo JH, Mauldin JP, et al. Cytotoxicity of human recombinant arginase I (Co)-PEG5000 in the presence of supplemental L-citrulline is dependent on decreased argininosuccinate synthetase expression in human cells. Anticancer Drugs. 2012;23(1):51-64.

18. Savaraj N, You M, Wu C, Wangpaichitr M, Kuo MT, Feun LG. Arginine deprivation, autophagy, apoptosis (AAA) for the treatment of melanoma. Curr Mol Med. 2010;10(4):405-412.

19. Mauldin JP, Zeinali I, Kleypas K, et al. Recombinant human arginase toxicity in mice is reduced by citrulline supplementation. Transl Oncol. 2012;5(1):26-31.

20. Das K, Butler GH, Kwiatkowski V, Clark AD Jr, Yadav P, Arnold E. Crystal structures of arginine deiminase with covalent reaction intermediates; implications for catalytic mechanism. Structure. 2004;12(4):657-667.

21. Takaku H, Matsumoto M, Misawa S, Miyazaki K. Anti-tumor activity of arginine deiminase from Mycoplasma argini and its growth-inhibitory mechanism. Jpn J Cancer Res. 1995;86(9):840-846.

22. Ensor CM, Holtsberg FW, Bomalaski JS, Clark MA. Pegylated arginine deiminase (ADI-SS PEG20,000 mw) inhibits human melanomas and hepatocellular carcinomas in vitro and in vivo. Cancer Res. 2002;62(19):5443-5450.

23. Holtsberg FW, Ensor CM, Steiner MR, Bomalaski JS, Clark MA. Poly(ethylene glycol) (PEG) conjugated arginine deiminase: effects of PEG formulations on its pharmacological properties. JControl Release. 2002;80(1-3):259-271.

24. Takaku H, Takase M, Abe S, Hayashi H, Miyazaki K. In vivo anti-tumor activity of arginine deiminase purified from Mycoplasma arginini. Int J Cancer. 1992;51(2):244-249.

25. Feun L, Savaraj N. Pegylated arginine deiminase: a novel anticancer enzyme agent. Expert Opin Investig Drugs. 2006;15(7):815-822.

26. Kuo MT, Savaraj N, Feun LG. Targeted cellular metabolism for cancer chemotherapy with recombinant arginine-degrading enzymes. Oncotarget. 2010;1(4):246-251.

27. Lam TL, Wong GK, Chow HY, et al. Recombinant human arginase inhibits the in vitro and in vivo proliferation of human melanoma by inducing cell cycle arrest and apoptosis. Pigment Cell Melanoma Res. 2011;24(2):366-376.

28. Stone EM, Glazer ES, Chantranupong L, et al. Replacing $\mathrm{Mn}(2+)$ with $\mathrm{Co}(2+)$ in human arginase i enhances cytotoxicity toward 1-arginine auxotrophic cancer cell lines. ACS Chem Biol. 2010;5(3):333-342.

29. Glazer ES, Stone EM, Zhu C, Massey KL, Hamir AN, Curley SA. Bioengineered human arginase I with enhanced activity and stability controls hepatocellular and pancreatic carcinoma xenografts. Transl Oncol. 2011;4(3):138-146.

30. Feun LG, You M, Wu C, et al. Final results of phase II trial of pegylated arginine deiminase (ADI-PEG20) in metastatic melanoma (MM) [abstract]. J Clin Oncol. 2010;28(Suppl 15):8528.

31. Tsai WB, Aiba I, Lee SY, Feun L, Savaraj N, Kuo MT. Resistance to arginine deiminase treatment in melanoma cells is associated with induced argininosuccinate synthetase expression involving c-Myc/ HIF-1alpha/Sp4. Mol Cancer Ther. 2009;8(12):3223-3233.

32. Feun $\mathrm{L}$, You M, Wu CJ, et al. Arginine deprivation as a targeted therapy for cancer. Curr Pharm Des. 2008;14(11):1049-1057. 
33. You M, Savaraj N, Wu CJ, et al. Enhancing arginine deprivation therapy in melanoma by combining with cisplatin. Paper presented at: $101 \mathrm{~s}$ Annual Meeting of the American Association of Cancer Research; April 17-21, 2010; Washington, DC.

34. You M, Savaraj N, Wangpaichitr M, et al. The combination of ADIPEG20 and TRAIL effectively increases cell death in melanoma cell lines. Biochem Biophys Res Commun. 2010;394(3):760-766.

35. Vynnytska BO, Mayevska OM, Kurlishchuk YV, Bobak YP, Stasyk OV. Canavanine augments proapoptotic effects of arginine deprivation in cultured human cancer cells. Anticancer Drugs. 2011;22(2):148-157.

36. Vynnytska-Myronovska B, Bobak Y, Garbe Y, Dittfeld C, Stasyk OV, Kunz-Schughart LA. Single amino acid arginine starvation efficiently sensitizes cancer cells to canavanine treatment and irradiation. Int $J$ Cancer. 2012;130(9):2164-2175.

37. Szlosarek PW, Klabatsa A, Pallaska A, et al. In vivo loss of expression of argininosuccinate synthetase in malignant pleural mesothelioma is a biomarker for susceptibility to arginine depletion. Clin Cancer Res. 2006;12(23):7126-7131

38. Yoon CY, Shim YJ, Kim EH, et al. Renal cell carcinoma does not express argininosuccinate synthetase and is highly sensitive to arginine deprivation via arginine deiminase. Int J Cancer. 2007; 120(4):897-905.

39. Kim RH, Coates JM, Bowles TL, et al. Arginine deiminase as a novel therapy for prostate cancer induces autophagy and caspase-independent apoptosis. Cancer Res. 2009;69(2):700-708.

40. Noh EJ, Kang SW, Shin YJ, et al. Arginine deiminase enhances dexamethasone-induced cytotoxicity in human T-lymphoblastic leukemia CCRF-CEM cells. Int J Cancer. 2004;112(3):502-508.

41. Kobayashi E, Masuda M, Nakayama R, et al. Reduced argininosuccinate synthetase is a predictive biomarker for the development of pulmonary metastasis in patients with osteosarcoma. Mol Cancer Ther. 2010;9(3):535-544.
42. Curley SA, Bomalaski JS, Ensor CM, Holtsberg FW, Clark MA. Regression of hepatocellular cancer in a patient treated with arginine deiminase. Hepatogastroenterology. 2003;50(53):1214-1216.

43. Izzo F, Marra P, Beneduce G, et al. Pegylated arginine deiminase treatment of patients with unresectable hepatocellular carcinoma: results from phase I/II studies. J Clin Oncol. 2004;22(10):1815-1822.

44. Ascierto PA, Scala S, Castello G, et al. Pegylated arginine deiminase treatment of patients with metastatic melanoma: results from phase I and II studies. J Clin Oncol. 2005;23(30):7660-7668.

45. Thomsen LL, Miles DW. Role of nitric oxide in tumour progression: lessons from human tumours. Cancer Metastasis Rev. 1998;17(1): 107-118.

46. Vallance P, Leiper J. Blocking NO synthesis: how, where and why? Nat Rev Drug Discov. 2002;1(12):939-950.

47. Cooke JP. NO and angiogenesis. Atheroscler Suppl. 2003;4(4) 53-60.

48. Glazer ES, Piccirillo M, Albino V, et al. Phase II study of pegylated arginine deiminase for nonresectable and metastatic hepatocellular carcinoma. J Clin Oncol. 2010;28(13):2220-2226.

49. Yang TS, Lu SN, Chao Y, et al. A randomised phase II study of pegylated arginine deiminase (ADI-PEG 20) in Asian advanced hepatocellular carcinoma patients. Br J Cancer. 2010;103(7):954-960.

50. Yau CC, Chan P, Pang R, et al. A phase I study of recombinant human arginase I (rhArgI) for patients with advanced hepatocellular carcinoma [abstract]. J Clin Oncol. 2010;28:e13503.
Clinical Pharmacology: Advances and Applications

\section{Publish your work in this journal}

Clinical Pharmacology: Advances and Applications is an international, peer-reviewed, open access journal publishing original research, reports, reviews and commentaries on all areas of drug experience in humans. The manuscript management system is completely online and includes a very quick and fair peer-review system, which is all easy to use.

\section{Dovepress}

Visit http://www.dovepress.com/testimonials.php to read real quotes from published authors. 